\title{
Mesothelioma
}

\section{DOES MESOTHELIOMA RISK DECLINE AFTER 40 YEARS SINCE FIRST EXPOSURE? A POOLED ANALYSIS}

Alison Reid, ${ }^{1}$ Nicholas de Klerk, Geoffrey Berry, ${ }^{2}$ Corrado Magnani, ${ }^{3}$ Daniela Ferrante, ${ }^{3}$ William Musk, ${ }^{1}$ Enzo Merler ${ }^{4}{ }^{1}$ University of Western Australia, Perth, Australia; ${ }^{2}$ University of Sydney, Sydney, Australia; ${ }^{3}$ University of Eastern Piedmont, Piedmont, Italy; ${ }^{4}$ Veneto Mesothelioma Registry, Padua, Italy

\subsection{6/oemed-2011-100382.191}

Objectives The risk of malignant mesothelioma increases proportionally to the cumulative exposure and to the 3 rd or 4 th power of time since first exposed to asbestos. However, little is known about the risk of mesothelioma after more than 40 years since first exposure because most epidemiological studies do not have follow-up for such long periods of time.

Methods The data from 6 cohort studies of exposed workers (3 Italian railway workers cohorts, amosite workers' cohort, Eternit cohort, Wittenoom workers' cohort) and two cohorts with residential exposure (Wittenoom residents and Eternit wives cohort) has been pooled. A nested case control design matched cases and controls on calendar period and age. Conditional logistic regression and fractional polynomials were used to model the relationship between time since first exposure and risk of mesothelioma.

Results The combined data consisted of 22048 people with asbestos exposure (16 279 males, 5769 females), 649 cases of confirmed pleural mesothelioma (494 in males, 155 in females) and 142 cases of peritoneal mesothelioma (112 in males and 30 in females). Median time since first exposure was 38.2 years (IOR 26.5-46.6). Median duration of exposure was 2.61 years (IWR 0.5-15.0). The risk of pleural mesothelioma increased until 40 years since first exposure and then appeared to plateau. The peritoneal mesothelioma risk continued to increase. Conclusions Pooling the data from these cohort studies substantially increases the number of mesotheliomas for analysis. Women and men, pleural and peritoneal mesotheliomas, type of asbestos and location of exposure has been examined separately. 